\title{
Bright optical beams in weakly nonlocal media: Variational analysis
}

\author{
Kaloyan S. Bezuhanov, ${ }^{1}$ Alexander A. Dreischuh, ${ }^{1,3}$ and Wieslaw Krolikowski ${ }^{2}$ \\ ${ }^{1}$ Department of Quantum Electronics, Faculty of Physics, Sofia University, BG-1164 Sofia, Bulgaria \\ ${ }^{2}$ Laser Physics Centre, Research School of Physical Sciences and Engineering, Australian National University, \\ Canberra ACT 0200, Australia \\ ${ }^{3}$ Nonlinear Photonics Centre, Research School of Physical Sciences and Engineering, Australian National University, \\ Canberra ACT 0200, Australia
}

(Received 18 November 2007; published 11 March 2008)

\begin{abstract}
We employ a variational technique to describe the propagation of a Gaussian beam in a nonlinear, weakly nonlocal medium and derive the conditions for breathing soliton formation in both one and two transverse dimensions. The reduced one-dimensional results agree quantitatively with known exact nonlocal soliton solutions. We subsequently formulate a simple procedure for estimating the strength of a weak nonlocality and verify its applicability by direct numerical simulations.
\end{abstract}

DOI: 10.1103/PhysRevA.77.033825

PACS number(s): 42.65.Tg, 47.35.Fg, 45.10.Db

\section{INTRODUCTION}

The nonlinear response of a medium to an external action is spatially nonlocal when it depends not only on the action in the particular point, but also in the neighboring points. In a plasma the processes of heating and ionization are known to cause a nonlocal response [1]. The long lifetime of optically pumped atoms allows the atomic diffusion to transport the excitation away from the location of the laser [2]. Drift and/or diffusion of photoexcited carriers lead to a nonlocal response in photorefractive materials [3,4]. Spatial nonlocality in the nonlinear response is also present in Bose-Einstein condensates exhibiting long-range interparticle interactions $[5,6]$. This is also the case of nematic liquid crystals when the elastic intermolecular forces result in a refractive-index change extending well beyond the excitation region [7]. Finally, the heat conduction in materials with thermal nonlinearity leads to nonlocal changes in their refractive index [8-10]. Not that obvious, the analogy between parametric interaction in quadratic media and nonlocal cubic media $[11,12]$ has led to the understanding that quadratic and nonlocal Kerr solitons are equivalent [13]. Finally, recent works on light localization in soft matter such as colloidal nanosuspensions pointed out the inherently nonlocal character of nonlinearity [14].

It is worth mentioning that while typically spatial nonlocality is considered to be symmetric, transverse symmetry can be broken by anisotropic boundary conditions [10].

The nonlocal character of the nonlinearity can be often described in terms of the so-called nonlocal response function which determines the spatial extent of the nonlocality. The width of this function relative to the spatial scale of the wave intensity profile determines the degree (strength) of the nonlocality, $\gamma^{\prime}$ [15]. In the local limit, the response is just a $\delta$ function. In the highly nonlocal limit the beam evolution is described by a linear equation with the potential determined by the spatial structure of the response function $[16,17]$. While typically the evolution of a wave in a nonlocal medium has to be treated numerically in a few cases such as for the logarithmic nonlinearity, an exact analytical treatment is also possible [18]. Recent studies of nonlinear effects in non- local nonlinear media include exact analytical solutions for one-dimensional bright and dark solitons for weak nonlocality [19], and the prediction of the existence of stable ring vortex solitons in self-focusing media in the regime of strong nonlocality [20,21], as well as the demonstration of a dramatic effect of nonlocality on both interaction forces between dark spatial solitons [22] and soliton mobility in optical lattices [23]. Because of the crucial importance of nonlocality in many nonlinear phenomena, its characterization and influence on the beam propagation are of a great practical interest [24].

In this work we study theoretically the propagation of optical beams in weakly nonlocal nonlinear media using the variational technique. We show that this approximate method allows for a very accurate description of the formation of nonlocal solitons and their dynamics. Moreover, it also forms the basis of a simple experimental procedure for estimating the strength of weak nonlocality.

\section{THEORETICAL MODEL}

Let us consider a phenomenological model of a nonlinear Kerr-type medium, in which (see Fig. 1) the refractive index $n^{\prime}=n+\Delta n(I(x, y, z))$ changes with intensity $I(x, y, z)$ in a nonlocal way:

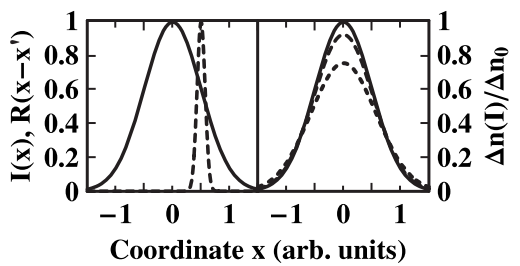

FIG. 1. Left: Gaussian intensity profile $I(x)$ (solid curve) and response function $R\left(x-x^{\prime}\right)$ (dashed curve) of respective widths $\sigma / \sqrt{2}$ and $w_{R}=0.1 \sigma / \sqrt{2}$. The weakly nonlocal limit corresponds to $w_{R} \ll \sigma$. Right: normalized distribution of the nonlinearly modulated refractive index for a local response (solid curve) and for degrees of nonlocality $w_{R} / \sigma=0.2$ and 0.4 (long- and short-dashed curves, respectively). 


$$
\begin{aligned}
\Delta n(I(x, y, z))= & \Delta n_{0} \int_{-\infty}^{\infty} R\left(x-x^{\prime}, y-y^{\prime}\right) I\left(x^{\prime}, y^{\prime}\right) \\
& \times d x^{\prime} d y^{\prime} / \int_{-\infty}^{\infty} R\left(x^{\prime}, y^{\prime}\right) d x^{\prime} d y^{\prime} .
\end{aligned}
$$

If the width $w_{R}$ of the real and symmetric response function $R\left(x^{\prime}, y^{\prime}\right)$ is much shorter than the radius $\sigma$ of the optical beam field amplitude, the nonlocality is weak $\left(\gamma^{\prime}=w_{R} / \sigma \ll 1\right)$ and one can use the expansion

$$
\begin{aligned}
I\left(x^{\prime}, y^{\prime}\right)= & I(x, y)+\frac{1}{2} \partial_{x x}^{2} I(x, y)\left(x-x^{\prime}\right)^{2}+\frac{1}{2} \partial_{y y}^{2} I(x, y)\left(y-y^{\prime}\right)^{2} \\
& +\partial_{x y}^{2} I(x, y)\left(x-x^{\prime}\right)\left(y-y^{\prime}\right)
\end{aligned}
$$

to obtain a simplified model of the nonlocal nonlinear correction to the refractive index $n$ :

$$
\begin{aligned}
\Delta n(I(x, y))= & \Delta n_{0}\left[I(x, y)+\frac{1}{2} \gamma_{x x} \partial_{x x}^{2} I(x, y)+\frac{1}{2} \gamma_{y y} \partial_{y y}^{2} I(x, y)\right. \\
& \left.+\gamma_{x y} \partial_{x y}^{2} I(x, y)\right] .
\end{aligned}
$$

The coefficients $\gamma_{\xi \xi}$ depend on $R(\xi, \eta)$ according to the relation

$$
\begin{aligned}
\gamma_{\xi \xi}= & \int_{-\infty}^{\infty} R\left(\xi-\xi^{\prime}, \eta-\eta^{\prime}\right)\left(\xi-\xi^{\prime}\right)^{2} d \xi^{\prime} d \eta^{\prime} / \int_{-\infty}^{\infty} R\left(\xi^{\prime}, \eta^{\prime}\right) \\
& \times d \xi^{\prime} d \eta^{\prime},
\end{aligned}
$$

and, for a Gaussian response, $\gamma_{\xi \xi}=w_{R \xi}^{2} / 2$. In a suitably chosen and rotated coordinate system the term

$$
\begin{aligned}
\gamma_{\xi \eta}= & \int_{-\infty}^{\infty} R\left(\xi-\xi^{\prime}, \eta-\eta^{\prime}\right)\left(\xi-\xi^{\prime}\right)\left(\eta-\eta^{\prime}\right) \\
& \times d \xi^{\prime} d \eta^{\prime} / \int_{-\infty}^{\infty} R\left(\xi^{\prime}, \eta^{\prime}\right) d \xi^{\prime} d \eta^{\prime}
\end{aligned}
$$

arising from the mixed derivative can be set to zero and will be further neglected. When the beamwidth decreases and becomes comparable to the characteristic response length of the medium, the nonlinearly modulated refractive index noticeable deviates from this in the local case (see Fig. 1). Therefore, nonlocality can have important consequences and has to be taken into account. It is worth mentioning that the weakly nonlocal nonlinearity has been recently identified as a stabilizing mechanism in the analysis of the nonlinear response of the colloidal suspension of dielectric nanospheres [14]. A similar model has been also investigated in the context of localized electron states in a discrete isotropic electronphonon lattice [25] and Bose Einstein condensate [26].

A term comprising $\partial^{2}\left(|E|^{2} E\right) / \partial \xi^{2}$ appears in the generalized nonlinear Schrödinger equation (NLSE) when derived [27] from Maxwell's equations by keeping the nonzero second derivative of the nonlinear polarization. This equation includes also terms reflecting a deviation from the paraxial approximation and the presence of a longitudinal component of the electric field [27]. Its simplified version contains the most essential term-namely, the one that accounts for the nonlinear diffraction. The notation "nonlinearly induced diffraction" was introduced for the first time in Refs. $[28,29]$ to stress the interplay between self-focusing and nonlocal response.

In the present analysis we adapt the heuristic approach of Hasegawa [30] initially used for deriving the master equation for information transfer in optical fibers (NLSE in the case of an instantaneous Kerr response) to the spatially nonlocal Kerr-type response. In this way we derive the twodimensional (2D) nonlocal NLSE (NNLSE) describing the evolution of the slowly varying electric-field amplitude $E(x, y, z)$ in weakly nonlocal media:

$$
\begin{aligned}
\frac{i}{k} \frac{\partial E}{\partial z} & +\frac{1}{2 k^{2}}\left(\frac{\partial^{2} E}{\partial x^{2}}+\frac{\partial^{2} E}{\partial y^{2}}\right) \\
& +\frac{\Delta n_{0}}{n}\left(|E|^{2}+\frac{\gamma_{x x}}{2} \frac{\partial^{2}|E|^{2}}{\partial x^{2}}+\frac{\gamma_{y y}}{2} \frac{\partial^{2}|E|^{2}}{\partial y^{2}}\right) E=0 .
\end{aligned}
$$

The Euler-Lagrange equation

$$
\frac{\partial}{\partial x} \frac{\partial \hat{L}}{\partial\left(\partial E^{*} / \partial x\right)}+\frac{\partial}{\partial y} \frac{\partial \hat{L}}{\partial\left(\partial E^{*} / \partial y\right)}+\frac{\partial}{\partial z} \frac{\partial \hat{L}}{\partial\left(\partial E^{*} / \partial z\right)}-\frac{\partial \hat{L}}{\partial E^{*}}=0
$$

is equivalent to the NNLSE in the sense of the Lagrange operator with a density $\hat{L}$ :

$$
\begin{aligned}
\hat{L}= & (i / 2 k)\left(E \partial_{z} E^{*}-E^{*} \partial_{z} E\right)+\left(1 / 2 k^{2}\right)\left(\left|\partial_{x} E\right|^{2}+\left|\partial_{y} E\right|^{2}\right) \\
& -\left(\Delta n_{0} / 2 n\right)\left\{|E|^{4}-\left(\gamma_{x x} / 2\right)\left(\partial_{x}|E|^{2}\right)^{2}-\left(\gamma_{y y} / 2\right)\left(\partial_{y}|E|^{2}\right)^{2}\right\} .
\end{aligned}
$$

In essence, the variational approach requires selection of a trial function $E=E\left(x, y, q_{i}(z)\right)$, depending not only on the transverse coordinates $x$ and $y$, but also on $z$ by suitable variational variables (functions) $q_{i}(z)$ [31]. Substituting the trial function in $\hat{L}$ and integrating the result over the transverse coordinates one gets the Lagrangian $\langle\hat{L}\rangle$ :

$$
\left\langle\hat{L}\left(\dot{q}_{i}(z), q_{i}(z)\right)\right\rangle=\iint \hat{L}\left(x, y, \dot{q}_{i}(z), q_{i}(z) d x d y,\right.
$$

which has to satisfy the system of Lagrange equations

$$
\frac{d}{d z} \frac{\partial\left\langle\hat{L}\left(\dot{q}_{i(z)}, q_{i(z)}\right)\right\rangle}{\partial \dot{q}_{i(z)}}-\frac{\partial\left\langle\hat{L}\left(\dot{q}_{i(z)}, q_{i(z)}\right)\right\rangle}{\partial q_{i(z)}}=0 .
$$

Overdots denote derivatives with respect to the propagation coordinate $z$. The result of the variational procedure is a system of ordinary differential equations for the variational variables $q_{i}$.

\section{TWO-DIMENSIONAL EVOLUTION}

Let us analyze a Gaussian beam with a slowly varying electric-field amplitude 


$$
\begin{aligned}
E(x, y, z)= & A(z) \exp \left\{-\frac{x^{2}}{\sigma_{x}^{2}(z)}-\frac{y^{2}}{\sigma_{y}^{2}(z)}+i \frac{k}{2}\left[\vartheta_{x}(z) x^{2}+\vartheta_{y}(z) y^{2}\right]\right. \\
& +i \varphi(z)\},
\end{aligned}
$$

where $\sigma_{\xi}$ and $\vartheta_{\xi}$ are the beamwidths and wave-front curvatures along the two coordinate system axes, $k$ is the wave number, and $\varphi$ is phase accumulated along the propagation path length. Following the variational procedure, we get

$$
\begin{aligned}
\langle\hat{L}\rangle= & (\pi / 2) \sigma_{x} \sigma_{y} A^{2}\left[\left(\dot{\vartheta}_{x} \sigma_{x}^{2}+\dot{\vartheta}_{y} \sigma_{y}^{2}+\vartheta_{x}^{2} \sigma_{x}^{2}+\vartheta_{y}^{2} \sigma_{y}^{2}\right) / 8\right. \\
& +\left(\sigma_{x}^{-2}+\sigma_{y}^{-2}\right) /\left(2 k^{2}\right)-\Delta n_{0}\left(1-\gamma_{x x} \sigma_{x}^{-2}-\gamma_{y y} \sigma_{y}^{-2}\right) \\
& \left.\times A^{2} /(4 n)+\dot{\varphi} / k\right] .
\end{aligned}
$$

Subsequently, we derive the following system of ordinary differential equations (ODEs) for the variational variables:

$$
\begin{gathered}
\dot{\sigma}_{x}=\vartheta_{x} \sigma_{x}, \\
\dot{\vartheta}_{x}=-\vartheta_{x}^{2}+4 /\left(k^{2} \sigma_{x}^{4}\right)-\left(\Delta n_{0} / n\right) A^{2} \sigma_{x}^{-2}\left(1-3 \gamma_{x x} \sigma_{x}^{-2}-\gamma_{y y} \sigma_{y}^{-2}\right), \\
\dot{\sigma}_{y}=\vartheta_{y} \sigma_{y}, \\
\dot{\vartheta}_{y}=-\vartheta_{y}^{2}+4 /\left(k^{2} \sigma_{y}^{4}\right)-\left(\Delta n_{0} / n\right) A^{2} \sigma_{y}^{-2}\left(1-3 \gamma_{y y} \sigma_{y}^{-2}-\gamma_{x x} \sigma_{x}^{-2}\right), \\
\dot{\varphi}=(-1 / k)\left(\sigma_{x}^{-2}+\sigma_{y}^{-2}\right)+k\left(\Delta n_{0} / n\right) A^{2}\left[(3 / 4)-\gamma_{x x} \sigma_{x}^{-2}\right. \\
\left.-\gamma_{y y} \sigma_{y}^{-2}\right],
\end{gathered}
$$$$
(1 / k)(d / d z)\left[(\pi / 2) \sigma_{x} \sigma_{y} A^{2}\right]=0 .
$$

The last equation reflects the energy conservation $P=P_{0}$ $=(\pi / 2) A^{2} \sigma_{x} \sigma_{y}=(\pi / 2) A_{0}^{2} \sigma_{x 0} \sigma_{y 0}$. Making use of this law, after routine transformations, one gets two second-order equations for the evolution of the beam's transverse dimensions:

$$
\begin{aligned}
& \ddot{\sigma}_{x}=4 /\left(k^{2} \sigma_{x}^{3}\right)-2\left(\Delta n_{0} / n\right) P_{0}\left(1-3 \gamma_{x x} \sigma_{x}^{-2}-\gamma_{y y} \sigma_{y}^{-2}\right) /\left(\pi \sigma_{x}^{2} \sigma_{y}\right), \\
& \ddot{\sigma}_{y}=4 /\left(k^{2} \sigma_{y}^{3}\right)-2\left(\Delta n_{0} / n\right) P_{0}\left(1-\gamma_{x x} \sigma_{x}^{-2}-3 \gamma_{y y} \sigma_{y}^{-2}\right) /\left(\pi \sigma_{x} \sigma_{y}^{2}\right) .
\end{aligned}
$$

The initial conditions for solving this system are $\sigma_{\xi}\left(z=z_{0}\right)$ $=\sigma_{\xi 0}$ and $\dot{\sigma}_{\xi}\left(z=z_{0}\right)=\vartheta_{\xi 0} \sigma_{\xi 0}$ for $\xi=x, y$.

Equations (13) admit solutions in the form of elliptic 2D solitons if $\sigma_{x 0}=\sigma_{x s}, \sigma_{y 0}=\sigma_{y s}$, and $\dot{\sigma}_{x} \equiv \dot{\sigma}_{y} \equiv 0$-i.e., $\vartheta_{x} \equiv \vartheta_{y}$ $\equiv \vartheta_{x 0} \equiv \vartheta_{y 0}=0$. Since $\ddot{\sigma}_{x} \equiv 0$ implies that

$$
k^{2} \frac{\Delta n_{0}}{n}\left(1-3 \frac{\gamma_{\xi \xi}}{\sigma_{\xi s}^{2}}-\frac{\gamma_{\eta \eta}}{\sigma_{\eta s}^{2}}\right) \frac{\sigma_{\xi s}}{\sigma_{\eta s}} P_{s}=2 \pi
$$

for $\xi, \eta=x, y(\xi \neq \eta)$, the last system is equivalent to

$$
\sigma_{\xi s}^{2}=\frac{4+3 k^{2}\left(\gamma_{\xi \xi}+\gamma_{\eta \eta}\right) I_{s} \Delta n_{0} / n+2 \gamma_{\xi \xi} \gamma_{\eta \eta}\left(k^{2} I_{s} \Delta n_{0} / n\right)^{2}}{k^{2}\left(1+k^{2} \gamma_{\eta \eta} \Delta n_{0} / 2 n\right) I_{s} \Delta n_{0} / n} .
$$

Equation (15) and the conservation law $P_{s}=(\pi / 2) \sigma_{x s} \sigma_{y s} I_{s}$ define parametrically a curve in 3D space. As seen in Fig. 2,

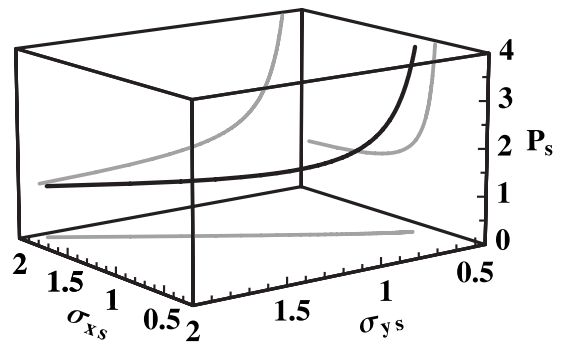

FIG. 2. Parametric plot $\sigma_{x s}=\sigma_{x s}\left(I_{s}\right), \sigma_{y s}=\sigma_{y s}\left(I_{s}\right)$, and $P_{s}$ $=P_{s}\left(I_{s}\right)$. The soliton power and the beam radii are normalized to their local values. Medium anisotropy $\gamma_{x x} / \gamma_{y y}=2$.

the increase of the beam power results in an asymmetric beam shrinking (and an increase of the ellipticity) at a nonnegligible anisotropy of the nonlocality $\left(\gamma_{x x} / \gamma_{y y}=2\right.$ in our example). Again from Eq. (15) one can see that when the nonlocality acts along one of the coordinates only (e.g., $\gamma_{y y}$ $=0$ ), it is possible to create an elliptic soliton with a maximal ellipticity:

$$
\sigma_{y s}^{2} / \sigma_{x s}^{2}=\left(1-3 \gamma_{x x} / \sigma_{x s}^{2}\right) /\left(1-\gamma_{x x} / \sigma_{x s}^{2}\right) .
$$

Unfortunately a quantitative comparison with the results in Ref. [10] is not possible. The measured [10] soliton ellipticity indicates a lower limit of the strength of the nonlocality $\gamma_{\xi \xi}^{\prime} \sim 0.3$ which is well beyond the range allowed by our approach.

Symmetry in two dimensions means that $\gamma_{x x}=\gamma_{y y}=\gamma$, $\sigma_{x 0}=\sigma_{y 0}=\sigma_{0}$, and $\vartheta_{x 0}=\vartheta_{y 0}=\vartheta_{0}$. The beam radius $\sigma(z)$ $\equiv \sigma_{x}(z) \equiv \sigma_{y}(z)$ evolves according to

$$
\ddot{\sigma}=\left[1 /\left(k^{2} \sigma^{3}\right)\right]\left[4-2 k^{2}\left(\Delta n_{0} / n\right)\left(1-4 \gamma / \sigma^{2}\right) P_{0} / \pi\right],
$$

and the soliton power $P_{s}$ becomes

$$
P_{s}=\left(\frac{\pi}{2}\right) \frac{4 n}{k^{2} \Delta n_{0}\left(1-4 \gamma / \sigma_{s}^{2}\right)} .
$$

One can see that symmetric nonlocality imposes a lower limit $\sigma_{s, \min }^{2 \mathrm{D}}$ to the minimal beamwidth, $\sigma_{s}>\sigma_{s, \min }^{2 \mathrm{D}}=2 \sqrt{\gamma}$.

Equations (13) are equivalent to those describing the motion of a classical particle with coordinates $\left(\sigma_{x}, \sigma_{y}\right)$ and velocities $\left(\vartheta_{x} \sigma_{x}, \vartheta_{y} \sigma_{y}\right)$ in a potential $U$ with a kinetic energy $T$ (see Fig. 3), where

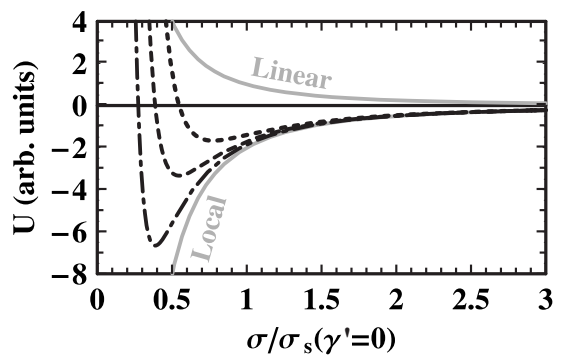

FIG. 3. Two-dimensional case: potential $U$ vs $\sigma_{s}$ normalized to the soliton beam radius $\sigma_{s}\left(\gamma^{\prime}=0\right)$. Dash-dotted curve, $\gamma^{\prime}=0.025$; dashed curve, $\gamma^{\prime}=0.05$; and dotted curve, $\gamma^{\prime}=0.1$. Beam power $P_{0}=3 P_{s}\left(\gamma^{\prime}=0\right)$. 


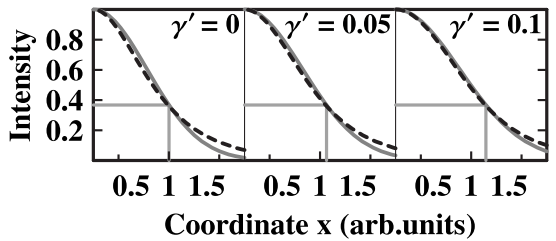

FIG. 4. Intensity profiles of $1 \mathrm{D}$ nonlocal spatial solitons for different values of the parameter $\gamma^{\prime}=\gamma_{x x} / \sigma_{x s}^{2}(\gamma=0)$. Solid curves, variational results; dashed curves, exact analytical solutions (see Ref. [19]).

$$
\begin{aligned}
& U=\left(2 / k^{2}\right)\left(\sigma_{x}^{-2}+\sigma_{y}^{-2}\right)-2\left(\Delta n_{0} / n\right) \\
& \times P_{0}\left(1-\gamma_{x x} \sigma_{x}^{-2}-\gamma_{y y} \sigma_{y}^{-2}\right) /\left(\pi \sigma_{x} \sigma_{y}\right), \\
& T=\left(\dot{\sigma}_{x}^{2}+\dot{\sigma}_{y}^{2}\right) / 2=\left(\vartheta_{x}^{2} \sigma_{x}^{2}+\vartheta_{y}^{2} \sigma_{y}^{2}\right) .
\end{aligned}
$$

The coordinates of the global minimum of the potential $U$ correspond to the soliton radii, whereas the initial negative total energy $\left(U_{0}+T_{0}<0\right)$ limits the region of possible breathing solitons (which, loosely speaking, periodically recover their intensity profiles). More precisely, the inequality

$$
4-2 k^{2} \frac{\Delta n_{0} P_{0}}{n \pi}\left(1-2 \frac{\gamma}{\sigma_{0}^{2}}\right)+k^{2} \vartheta_{0}^{2} \sigma_{0}^{4}<0
$$

is the condition for forming a breathing soliton in the symmetric $2 \mathrm{D}$ case.

\section{ONE-DIMENSIONAL EVOLUTION}

Considering the nonlinear propagation of an onedimensional Gaussian beam $\left(E(x, y, z)=A(z) \exp \left[-x^{2} / \sigma_{x}^{2}(z)\right.\right.$ $\left.\left.+i k \vartheta_{x}(z) x^{2} / 2+i \varphi(z)\right]\right)$ in a weakly nonlocal nonlinear medium and following the procedure described earlier, one gets the following reduced system of ODEs for the variational variables:

$$
\begin{gathered}
\dot{\sigma}_{x}=\vartheta_{x} \sigma_{x}, \\
\dot{\vartheta}_{x}=-\vartheta_{x}^{2}+4 /\left(k^{2} \sigma_{x}^{4}\right)-\left(\sqrt{2} \Delta n_{0} / n\right) A^{2} \sigma_{x}^{-2}\left(1-3 \gamma_{x x} \sigma_{x}^{-2}\right), \\
\dot{\varphi}=(-1 / k) \sigma_{x}^{-2}+k\left(\sqrt{2} \Delta n_{0} / n\right) A^{2}\left[(3 / 4)-\gamma_{x x} \sigma_{x}^{-2}\right], \\
k(d / d z)\left(\sqrt{\pi / 2} \sigma_{x} A^{2}\right)=0 .
\end{gathered}
$$

Accounting for the conservation of the beam power $P_{0}$ we arrive at the second-order ODE describing the changes in the beamwidth $\sigma_{x}$ :

$$
\ddot{\sigma}_{x}=4 /\left(k^{2} \sigma_{x}^{3}\right)-\left(2 \Delta n_{0} / n\right)\left[P_{0} /\left(\sqrt{\pi} \sigma_{x}^{2}\right)\right]\left(1-3 \gamma_{x x} \sigma_{x}^{-2}\right) .
$$

This equation admits a soliton solution when $\sigma_{x 0}=\sigma_{x s}$ =const, $\dot{\sigma}_{x} \equiv 0$, and $\ddot{\sigma}_{x} \equiv 0$ under the plane-wave condition $\vartheta_{x} \equiv \vartheta_{x 0}=0$. The analytical result for the soliton power $P_{s}$,

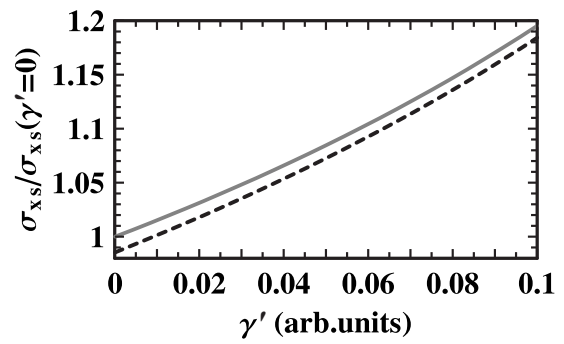

FIG. 5. Soliton width $\sigma_{x s}$ normalized to its respective value $\sigma_{x s}\left(\gamma^{\prime}=0\right)$ in the local case as a function of the degree of nonlocality, $\gamma^{\prime}=\gamma_{x x} / \sigma_{x s}^{2}(\gamma=0)$. Solid and dashed curves: variational and exact analytical [19] results for $P=P_{s}$.

$$
P_{s}=\frac{2 \sqrt{\pi}}{k^{2} \sigma_{x s}\left(\Delta n_{0} / n\right)\left(1-3 \gamma_{x x} / \sigma_{x s}^{2}\right)},
$$

indicates that in each particular case of nonlinear and nonlocal parameters $\Delta n_{0}$ and $\gamma_{x x}$ the soliton radius $\sigma_{x s}$ has to exceed the minimal value of $\sigma_{x s, \min }^{1 \mathrm{D}}=\sqrt{3 \gamma_{x x}}$. In agreement with intuition, the higher the nonlocality, the larger the minimal achievable width of both the $1 \mathrm{D}$ and $2 \mathrm{D}$ beams. For the same degree of nonlocality the difference in the dimensionality is reflected in the ratio $\sigma_{s, \text { min }}^{2 \mathrm{D}} / \sigma_{x s, \text { min }}^{1 \mathrm{D}}=2 / \sqrt{3}$.

At this point it is important to compare the variational results with the exact 1D theory of Ref. [19]. Figure 4 clearly shows that the Gaussian trial function used here approximates reasonably well the shape of the actual nonlocal soliton. Qualitatively, the deviation decreases with higher nonlocality. Since the nonlocality smooths out the refractive index profile, the soliton width increases monotonically with $\gamma^{\prime}$. In Fig. 5 we present this dependence and compare it with exact analytical results [19]. As is evident, the agreement is fairly good and the relative differences between the beam radii remain below 2\%. In Fig. 6 we show the relation between soliton power and degree of nonlocality [see Eqs. (18) and (23)]. Shaded regions mark the respective parameter ranges in which the combined action of linear and nonlinear diffraction cannot be canceled by the beam's self-focusing. Therefore, no solitons can be formed there. The interval, in

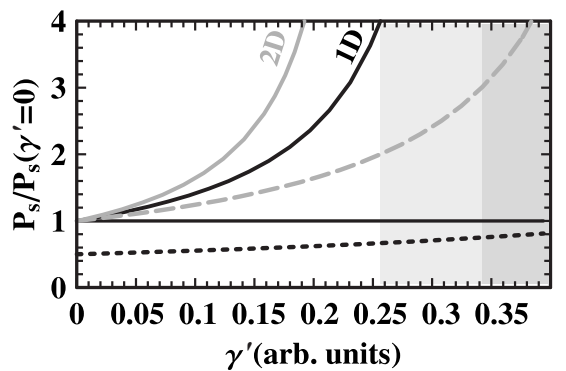

FIG. 6. Rise of the normalized soliton power $P_{s} / P_{s}\left(\gamma^{\prime}=0\right)$ with increasing the nonlocality $\gamma^{\prime}$. Solid curves: results from Eqs. (23) and (18) in the 1D and 2D cases, respectively. Solid horizontal line: local medium. Minimal power levels needed to form breathing solitons in 1D (dotted curve) and 2D (dashed curve). Light and dark shaded area: nonsoliton regions of a total domination of the intensity-dependent diffraction. 
which the weak nonlocality approximation and the model NNLSE hold, spans up to $\gamma^{\prime} \leq 0.1$. The minimal power levels needed to form breathing solitons in the 1D (dotted curve) and 2D (dashed curve) cases correspond to inequalities (26) and (20), respectively. The dotted curve indicates that the 1D breathing soliton can be excited at power levels well below the level needed to form a 1D local spatial soliton (solid horizontal line). The solid curves show that the soliton power increases with nonlocality $\gamma^{\prime}=\gamma_{x x} / \sigma_{x s}^{2}(\gamma=0)$.

Integrating Eq. (22) ones one gets

$$
\begin{aligned}
\dot{\sigma}_{x}= & -\frac{4}{k^{2} \sigma_{x}^{2}}+\frac{4 \Delta n_{0} P_{0}}{\sqrt{\pi} n \sigma_{x}}\left(1-\frac{\gamma_{x x}}{\sigma_{x}^{2}}\right)+\frac{4}{k^{2} \sigma_{x 0}^{2}} \\
& -\frac{4 \Delta n_{0} P_{0}}{\sqrt{\pi} n \sigma_{x 0}}\left(1-\frac{\gamma_{x x}}{\sigma_{x 0}^{2}}\right)+\vartheta_{x 0}^{2} \sigma_{x 0}^{2},
\end{aligned}
$$

which allows one, in the case of a nonstationary propagation along the nonlinear medium, to calculate the extrema $\sigma_{x, \text { extr }}$ of the beamwidth $\sigma_{x}(z)$ under the initial condition $\dot{\sigma}_{x}=0$ by

$$
\begin{aligned}
{[1} & \left.-k^{2} \frac{\Delta n_{0} P_{0} \sigma_{x 0}}{n \sqrt{\pi}}\left(1-\frac{\gamma_{x x}}{\sigma_{x 0}^{2}}\right)+\frac{k^{2} \vartheta_{x 0}^{2} \sigma_{x 0}^{4}}{4}\right] \frac{\sigma_{x, \text { extr }}^{3}}{\sigma_{x 0}^{3}} \\
& +k^{2} \frac{\Delta n_{0} P_{0} \sigma_{x 0}}{n \sqrt{\pi}}\left(\frac{\sigma_{x, \text { extr }}^{2}}{\sigma_{x 0}^{2}}-\frac{\gamma_{x x}}{\sigma_{x 0}^{2}}\right)-\frac{\sigma_{x, \text { extr }}}{\sigma_{x 0}}=0 .
\end{aligned}
$$

This equation has two real and positive solutions corresponding to minimal and maximal physical beam radii $\sigma_{x, \min }$ and $\sigma_{x, \text { max }}$, respectively, only if

$$
1-k^{2} \frac{\Delta n_{0} P_{0} \sigma_{x 0}}{n \sqrt{\pi}}\left(1-\frac{\gamma_{x x}}{\sigma_{x 0}^{2}}\right)+\frac{k^{2} \vartheta_{x 0}^{2} \sigma_{x 0}^{4}}{4}<0 .
$$

Since $\sigma_{x}(z)$ is allowed to oscillate between $\sigma_{x, \text { min }}$ and $\sigma_{x, \max }$, inequality (26) [the 1D analog of Eq. (20)] is the condition to create a breathing solitary wave. The soliton is a particular case of a breather for which $\sigma_{x s}=\sigma_{x, \min }=\sigma_{x, \max }$. It is worth noting that Eq. (26) can be obtained from the condition of a negative total energy $(U+T<0)$ with a potential $U$ [corresponding to Eq. (22)] and kinetic energy $T$ :

$$
\begin{gathered}
U=2 /\left(k^{2} \sigma_{x}^{2}\right)-2\left(\Delta n_{0} / n\right) P_{0}\left(1-\gamma_{x x} \sigma_{x}^{-2}\right) /\left(\pi \sigma_{x}\right), \\
T=\dot{\sigma}_{x}^{2} / 2=\vartheta_{x}^{2} \sigma_{x}^{2} .
\end{gathered}
$$

In Fig. 7 we show the evolution of the beam radius $\sigma_{x}$ along the weakly nonlocal $\left(\gamma^{\prime}=0.05\right) 1 \mathrm{D}$ nonlinear medium. The radius $\sigma_{x}$ of the beam is normalized to that of the soliton beam $\sigma_{x s}$ (horizontal dashed line), and its evolution is followed up to 16 Rayleigh diffraction lengths. Initial beam focusing is denoted with $\vartheta<0$. The oscillating solid curve for $\vartheta=0$ corresponds to $\sigma_{x 0}=0.8 \sigma_{x s}$. The appended grayscale image of the 1D beam intensity clearly shows periodic beam broadening and contraction after propagation path length $z$ $\approx 6.2 L_{D}$. In an agreement with intuition, a gradually broader $\left(\sigma_{x 0}=2 \sigma_{x s}\right)$ and initially focused $(\vartheta>0)$ bright beam also undergoes focusing until the combined action of diffraction and nonlocality prevails. The shaded area in the graph denotes region not accessible for soliton formation according to inequality (26). Long-dashed and short-dashed steep curves

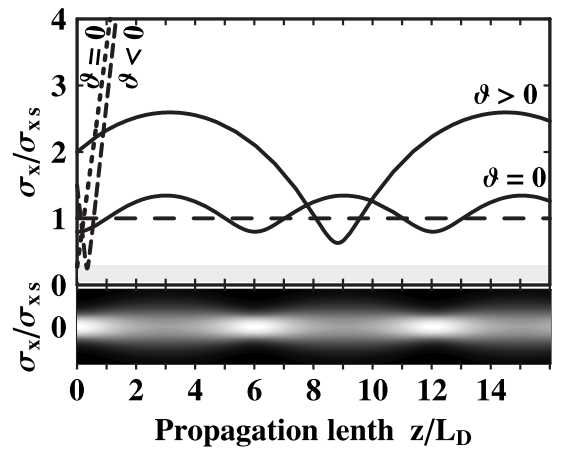

FIG. 7. Graph: evolution of the normalized beam radius along the nonlocal nonlinear medium with $(\vartheta \neq 0)$ and without $(\vartheta=0)$ initial beam focusing and defocusing. $\gamma^{\prime}=0.05$. Image: breathing soliton with an initial radius $\sigma_{s}=0.8 \sigma_{x s}$ and an initial plane wave front $(\vartheta=0)$. See text for details.

show the evolution of an initially unfocused narrow input beam $\left(\vartheta=0, \sigma_{x 0}=0.27 \sigma_{x s}\right)$ and a wide focused beam $\left(\sigma_{x 0}\right.$ $=1.5 \sigma_{x s}, \vartheta<0$ ) starting from (respectively, penetrating in) the forbidden region. The beam power is kept $P=P_{s}$. As seen, beams can penetrate in the forbidden region only once thereafter starting to continuously diverge.

\section{METHOD FOR ESTIMATING THE STRENGTH OF THE NONLOCALITY}

In the reminder of this work we will show that if breathing solitary waves are excited in a weakly nonlocal medium, it is possible to estimate the strength of the nonlocality.

\section{A. One-dimensional case}

Let us rewrite Eq. (25) in more convenient form

$$
\begin{array}{r}
\frac{n}{\Delta n_{0}} \frac{\sigma_{x, \text { extr }}}{\sigma_{x 0}}\left[\frac{1-\left(1+k^{2} \vartheta_{x 0}^{2} \sigma_{x 0}^{4} / 4\right) \sigma_{x, \text { extr }}^{2} / \sigma_{x 0}^{2}}{k^{2} P_{0} \sigma_{x 0} / \sqrt{\pi}}\right] \\
+\frac{\gamma_{x x}}{\sigma_{x 0}^{2}}\left(1-\frac{\sigma_{x, \text { extr }}^{3}}{\sigma_{x 0}^{3}}\right)=\frac{\sigma_{x, \text { extr }}^{2}}{\sigma_{x 0}^{2}}\left(1-\frac{\sigma_{x, \text { extr }}}{\sigma_{x 0}}\right),
\end{array}
$$

from which the linear relation between $1 / \Delta n_{0}$ and $\gamma_{x x}$ is transparent. At known initial conditions (beam power $P_{0}$, beam radius $\sigma_{x 0}$, and wave-front curvature $\vartheta_{x 0}$ ) one has to measure two extremal beamwidths $\sigma_{x, \text { extr }}$. Since this relation is fulfilled in all situations in which there is at least one real positive value of $\sigma_{x, \text { extr }}$ (if unique, it is a minimum), varying the accessible initial parameters one can measure the corresponding values of $\sigma_{x, \text { extr }}$ and calculate the ratio between the extremal and the initial beamwidths in the $j$ th measurement $\left(\Sigma_{j}=\sigma_{x, \text { extr }}^{j} / \sigma_{x 0}^{j}\right)$. Thereafter by solving a determined system of linear equations for each pair of extrema,

$$
\frac{n}{\Delta n_{0}} \Sigma_{j} \frac{1-\left(1+k^{2} \vartheta_{x 0 j}^{2} \sigma_{x 0 j}^{4} / 4\right) \Sigma_{j}^{2}}{k^{2} P_{0 j} \sigma_{x 0 j} / \sqrt{\pi}}+\frac{\gamma_{x x}}{\sigma_{x 0 j}^{2}}\left(1-\Sigma_{j}^{3}\right)=\Sigma_{j}^{2}\left(1-\Sigma_{j}\right),
$$

one can retrieve $\Delta n_{0}$ and $\gamma_{x x}$. 


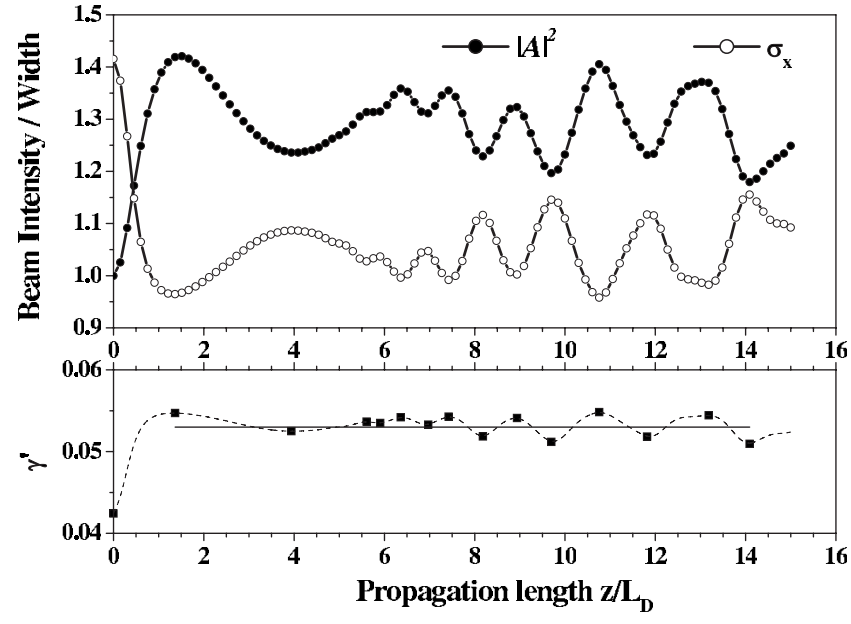

FIG. 8. Numerical solution of the NNLSE for nonlocality strength $\gamma^{\prime}=0.05$ (upper graph) and retrieved values of $\gamma^{\prime}$ (lower graph). See text for details.

In the particular case of an initial planar wave front $\left(\vartheta_{x 0}=0\right)$ one can prove that one of the values of $\sigma_{x, \text { extr }}$ corresponds to the initial beamwidth $\left(\sigma_{x, \text { extr }}=\sigma_{x 0}\right)$ and Eqs. (28) and (29) are trivial. The second value is related to the extremum limiting the deviation of the width of the breathing soliton from the initial one. In this case $\left(\vartheta_{x 0}=0\right)$ one has to measure in the experiment the corresponding extrema for two different initial beam powers (or beam radii) and solve Eq. (29).

In order to verify these predictions we treated the grayscale image shown in Fig. 7 as an experimental one. Since for the planar initial conditions the minima are not informative (trivial solutions), we took the value of $\Delta n_{0}$ from the model parameters. Interpreting one pixel of the computed image shown in Fig. 7 as the detector spatial resolution, we deduced $\gamma^{\prime}=0.048$, in a close agreement with the value of 0.05 used in the actual simulation. Interpolating the quasiexperimental beam profiles, thus increasing twice the accuracy in determining $\sigma_{x, \max }$, the error in retrieving $\gamma^{\prime}$ is reduced to below $1 \%$.

As a next step we solved numerically the NNLSE [Eq. (5) in Ref. [19]] for a collimated input Gaussian beam and nonlocality strength $\gamma^{\prime}=0.1$ and 0.05 , as well as in the nonlinear local and linear cases. The last two simulations are necessary to calibrate the longitudinal scale and the conserved power $P_{0}$ and the soliton power $P_{s}\left(\gamma^{\prime}=0\right)$ in the local case. By using the explicit relation (23), Eq. (28) becomes quadratic in $\gamma_{x x}$ :

$$
\begin{aligned}
& \frac{2 \sigma_{x, \mathrm{extr}}}{\alpha \sigma_{\mathrm{sol}}\left(1-3 \gamma_{x x} / \sigma_{\mathrm{sol}}^{2}\right)}\left(1-\frac{\sigma_{x, \mathrm{extr}}}{\sigma_{x 0}}\right)+\gamma_{x x}\left(1+\frac{\sigma_{x, \mathrm{extr}}}{\sigma_{x 0}}+\frac{\sigma_{x, \mathrm{extr}}^{2}}{\sigma_{x 0}^{2}}\right) \\
& =\sigma_{x, \mathrm{extr}}^{2} .
\end{aligned}
$$

Here $\sigma_{0}, \sigma_{\text {sol }}$ and $\sigma_{x, \text { extr }}$ are the initial, local soliton, and extremal breathing soliton radii, respectively, and $\alpha$ $=P_{\text {sol }} / P_{0}$. The top graph in Fig. 8 shows the $1 \mathrm{D}$ evolution of the peak intensity and the radius of an initially Gaussian beam with $\sigma_{x 0}(z=0)=\sqrt{2}$ (at $1 / e$ intensity level). Retrieving $\sigma_{\text {sol }}=1.8$ and $\alpha=2.26$ from the numerical data and recalling that the definition of nonlocality strength in Ref. [19] is related to our notation by $\gamma^{\prime}=\gamma_{x x} /\left(2 \sigma_{x 0}^{2}\right)$, we calculated $\gamma^{\prime}$ for each extremum of the width of the breathing beam. Such found real and positive values are plotted in the bottom graph in Fig. 8. The dashed line is intended to guide the eye through the results, obtained for nonextremal values of $\sigma_{x}$. The straight line denotes the retrieved mean value of the degree of nonlocality, $\gamma^{\prime}=0.053$, close to the value of 0.05 used in the numerical simulation.

At the end we would like to note that if the input beam is focused $\left(\vartheta_{x 0} \neq 0\right)$ Eqs. (28) and (29) are informative when (i) the input beamwidth decreases until $\sigma_{x, \text { extr }}=\sigma_{x, \min }$ is reached and thereafter starts to diverge monotonically (see the steep curves in Fig. 7) or (ii) the breathing soliton is excited (see Fig. 7).

\section{B. Symmetric two-dimensional case}

Following the same approach, integrating Eq. (17) once, we obtain an ODE

$$
\begin{aligned}
\dot{\sigma}= & -\frac{4}{k^{2} \sigma^{2}}+\frac{2 \Delta n_{0} P_{0}}{\pi n \sigma^{2}}\left(1-2 \frac{\gamma}{\sigma^{2}}\right)+\frac{4}{k^{2} \sigma_{0}^{2}} \\
& -\frac{2 \Delta n_{0} P_{0}}{\pi n \sigma_{0}^{2}}\left(1-2 \frac{\gamma}{\sigma_{0}^{2}}\right)+\vartheta_{0}^{2} \sigma_{0}^{2},
\end{aligned}
$$

according to which the extrema of the beamwidth $\sigma=\sigma(z)$ are solutions of the equation

$$
\begin{gathered}
{\left[4-2 k^{2} \frac{\Delta n_{0} P_{0}}{n \pi}\left(1-2 \frac{\gamma}{\sigma_{0}^{2}}\right)+k^{2} \vartheta_{0}^{2} \sigma_{0}^{4}\right] \frac{\sigma_{\text {extr }}^{4}}{\sigma_{0}^{4}}} \\
+k^{2} \frac{2 \Delta n_{0} P_{0}}{n \pi}\left(\frac{\sigma_{\text {extr }}^{2}}{\sigma_{0}^{2}}-2 \frac{\gamma}{\sigma_{0}^{2}}\right)-4=0 .
\end{gathered}
$$

When the input beam has a plane wave front, $\sigma_{\text {extr }}$ is either equal to $\sigma_{0}$ or to $\sigma_{0} / \sqrt{2 \sigma_{0}^{2} / \sigma_{s}^{2}-1}$, where $\sigma_{s}$ is defined by Eq. (18) after the formal substitution $P_{s} \rightarrow P_{0}$. Rearranging the terms one gets an equation

$$
\begin{aligned}
& \frac{n}{\Delta n_{0}} \frac{\sigma_{\text {extr }}^{2}}{\sigma_{0}^{2}}\left[\frac{4-\left(4+k^{2} \vartheta_{0}^{2} \sigma_{0}^{4} / 4\right) \sigma_{\text {extr }}^{2} / \sigma_{0}^{2}}{2 k^{2} P_{0} / \pi}\right]+\frac{2 \gamma}{\sigma_{0}^{2}}\left(1-\frac{\sigma_{\text {extr }}^{4}}{\sigma_{0}^{4}}\right) \\
& \quad=\frac{\sigma_{\text {extr }}^{2}}{\sigma_{0}^{2}}\left(1-\frac{\sigma_{\text {extr }}^{2}}{\sigma_{0}^{2}}\right),
\end{aligned}
$$

which is well suited for the processing of experimental data as described in the previous $1 \mathrm{D}$ case. In a real experimental situation the medium absorption (if non-negligible) has to be measured independently and the beam power at a particular distance has to be rescaled. Let us mention again that all presented results are valid under the assumption of a weak nonlocality. Their applicability to particular experimental conditions has to be carefully clarified.

\section{CONCLUSION}

Starting from a phenomenological model of a spatially nonlocal Kerr-type nonlinear medium we heuristically de- 
rived a nonlocal nonlinear Schrödinger equation in the limit of weak nonlocality. In this equation and in the obtained variational results for Gaussian beam propagation the nonlocality appears in form of nonlinear diffraction. The interplay between the nonlinear diffraction and self-focusing is found to result in an increase of the power needed to form nonlocal spatial solitons. The 1D variational results agree qualitatively and quantitatively well with the exact nonlocal soliton solutions of Ref. [19]. The Gaussian trial function we used approximates reasonably well the shape of the nonlocal soliton. Our variational prediction for the soliton width as a function of the degree of nonlocality differs by less than $2 \%$ from the exact solution. The derived necessary conditions for generating breathing solitons enabled us to formulate a procedure for estimating the degree of weak nonlocality from the experimental data. Its feasibility is verified by analyzing numerical results obtained by directly solving the weakly nonlocal nonlinear Schrödinger equation.

\section{ACKNOWLEDGMENTS}

This work was partially supported by the National Science Fund (Bulgaria), under Contract No. F-1303/2003, and the Australian Research Council.
[1] A. Litvak, V. Mironov, G. Fraiman, and A. Yunakovskii, Sov. J. Plasma Phys. 1, 31 (1975).

[2] D. Suter and T. Blasberg, Phys. Rev. A 48, 4583 (1993).

[3] S. Gatz and J. Herrmann, Opt. Lett. 23, 1176 (1998).

[4] B. Crosignani, A. Degasperis, E. DelRe, P. Di Porto, and A. J. Agranat, Phys. Rev. Lett. 82, 1664 (1999).

[5] A. Parola, L. Salasnich, and L. Reatto, Phys. Rev. A 57, R3180 (1998).

[6] K. Goral, K. Rzazewski, and T. Pfau, Phys. Rev. A 61, 051601(R) (2000); S. Sinha and L. Santos, Phys. Rev. Lett. 99, 140406 (2007).

[7] M. Peccianti, C. Conti, and G. Assanto, Opt. Lett. 30, 415 (2005)

[8] S. Akhmanov, D. Krindach, A. Migulin, A. Sukhorukov, and R. Khokhlov, IEEE J. Quantum Electron. 4, 568 (1968).

[9] M. D. Iturbe Castillo, J. J. Sánchez-Mondragón, and A. Stepanov, Opt. Lett. 21, 1622 (1996).

[10] C. Rotschild, O. Cohen, O. Manela, M. Segev, and T. Carmon, Phys. Rev. Lett. 95, 213904 (2005); C. Rotschild, M. Segev, Z. Xu, Y. V. Kartashov, L. Torner, and O. Cohen, Opt. Lett. 31, 3312 (2006).

[11] I. V. Shadrivov and A. A. Zharov, J. Opt. Soc. Am. B 19, 596 (2002).

[12] C. Conti, M. Peccianti, and G. Assanto, Phys. Rev. Lett. 91, 073901 (2003); K. G. Makris, H. Sarkissian, D. N. Christodoulides, and G. Assanto, J. Opt. Soc. Am. B 22, 1371 (2005); M. Peccianti, C. Conti, and G. Assanto, Opt. Lett. 30, 415 (2005); A. Alberucci, M. Peccianti, G. Assanto, A. Dyadyusha, and M. Kaczmarek, Phys. Rev. Lett. 97, 153903 (2006).

[13] N. I. Nikolov, D. Neshev, O. Bang, and W. Z. Królikowski, Phys. Rev. E 68, 036614 (2003).

[14] C. Conti, G. Ruocco, and S. Trillo, Phys. Rev. Lett. 95,
183902 (2005); C. Conti, N. Ghofraniha, G. Ruocco, and S. Trillo, ibid. 97, 123903 (2006).

[15] J. Wyller, W. Królikowski, O. Bang, and J. J. Rasmussen, Phys. Rev. E 66, 066615 (2002).

[16] A. Snyder and J. Mitchell, Science 276, 1538 (1997).

[17] W. Zhong and Lin Yi, Phys. Rev. A 75, 061801(R) (2007).

[18] D. J. Mitchell and A. W. Snyder, J. Opt. Soc. Am. B 16, 236 (1999).

[19] W. Królikowski and O. Bang, Phys. Rev. E 63, 016610 (2000).

[20] D. Briedis, D. Petersen, D. Edmundson, W. Królikowski, and O. Bang, Opt. Express 13, 435 (2005).

[21] A. I. Yakimenko, Y. A. Zaliznyak, and Yu. Kivshar, Phys. Rev. E 71, 065603(R) (2005); V. M. Lashkin, Phys. Rev. A 75, 043607 (2007).

[22] A. Dreischuh, D. N. Neshev, D. E. Petersen, O. Bang, and W. Królikowski, Phys. Rev. Lett. 96, 043901 (2006).

[23] Z. Xu, Y. V. Kartashov, and L. Torner, Phys. Rev. Lett. 95, 113901 (2005).

[24] X. Hutsebaut, C. Cambournac, M. Haelterman, J. Beeckman, and K. Neyts, J. Opt. Soc. Am. B 22, 1424 (2005).

[25] L. Brizhik, A. Eremko, B. Piette, and W. J. Zakrzewski, Nonlinearity 16, 1481 (2003).

[26] V. M. Perez-Garcia, V. V. Konotop, and J. J. Garcia-Ripoll, Phys. Rev. E 62, 4300 (2000).

[27] B. A. Malomed, K. Marinov, D. I. Pushkarov, and A. Shivarova, Phys. Rev. A 64, 023814 (2001).

[28] A. D. Boardman, K. Marinov, D. I. Pushkarov, and A. Shivarova, Opt. Quantum Electron. 32, 49 (2000).

[29] K. Marinov, D. I. Pushkarov, and A. Shivarova, Phys. Scr. T84, 197 (2000).

[30] A. Hasegawa, Chaos 10, 475 (2000).

[31] D. Anderson, Phys. Rev. A 27, 3135 (1983). 